\section{Les chantiers de construction de la citadelle de Damas: méthodologie et résultats préliminaires}

\author{
Jean-Claude Bessac*, Marianne Boqvist** \\ * U.M.R. 5140 du C.N.R.S., Centre de Documentation de Lattes 390 \\ Avenue de Pérols 34970, Lattes, France \\ tél. 0033467156125 , fax 0033467225515 \\ tél/fax personnel: 00334668117 45, mail: j-c-bessac-cnrs@wanadoo.fr \\ ** Université de Paris IV, Institut Français d'Études Arabes de Damas (IFEAD) \\ B.P. 344, Damas, Syrie \\ tél.00963113538727, mail marianne.boqvist@sidaa.se
}

\section{Résumé}

De nombreuses publications sont parues sur l'architecture de la Citadelle e Damas tandis que les techniques de construction et l'organisation du travail n'ont jamais été étudiées en utilisant des méthodes archéologiques appropriées.

La présente étude traite de la «Salle à colonnes» qui relie la porte Est à la porte Nord de la Citadelle et du bâtiment sud-ouest, datés tous deux du xile et XIIIe siècles. L'étude vise la connaissance de la construction de ces bâtiments à partir d'une approche archéologique de l'architecture monumentale, fondée sur l'analyse de diverses données techniques dimensions, matériaux de construction (surtout la pierre), outils et leurs traces, techniques de taille de pierre (fonctionnelles et ornementales), voûtes, géométrie, structure des murs, marques lapidaires et éléments réutilisés.

Outre l'établissement d'une méthode de recherche, les résultats préliminaires de cette étude permettent d'aborder quelques aspects de l'organisation du travail dans la Citadelle de Damas. Cette analyse révèle en particulier une réutilisation des techniques de construction classiques et de l'organisation du travail antique avec, par exemple, la présence d'équipes de spécialistes travaillant sur des murs, des ouvertures, des arches et des voûtes. Ces équipes paraissent être très diverses et l'analyse de leur travail démontre qu'elles avaient une certaine autonomie dans le choix de leurs techniques de construction et de finition des pierres et de la maçonnerie.

Mots clés: Arc, Bossage, Construction militaire, Economie de la construction, Marque lapidaire, Taille de pierre, Technique de construction, Voûte

\section{Abstract \\ While much has been published on the architecture of the Citadel of Damascus, construction techniques and the work organisation have never been studied employing the appropriate archaeological methods. The present study deals with the salle à colonnes, connecting the eastern and the northern gate of the citadel and with the south-west building, both dated to the twelfth and thirteenth centuries. It seeks to understand the building from an archaeological approach to monumental architecture, based on the analysis of technical data including dimensions, building materials (principally stone), tools and toolmarks, stone cutting techniques (functional and ornamental), vaulting, geometry, structure of walls, masons marks and reused elements. Except for the establishment of a research method, the preliminary results of this research allow us to state some aspects of the organisation of labour in the citadel of Damascus. This comparison reveals in particular a re-utilisation of the classical building techniques and organisation of labour, with, for example, the presence of teams of specialists working on walls, openings, arches and vaults. These teams of workmen appear to be quite diverse and analysis of their work demonstrates that they had a certain autonomy in their choice of building techniques and in the finishing of stones and masonry.}

Key words: Arch, Bossed masonry, Construction economy, Construction technique, Masson's marks, Military construction, Site organization, Vault

\section{L'ÉTUDE DU BÂTI MONUMENTAL DE LA CITADELLE: SA PROBLÉMATIQUE, SA MÉTHODOLOGIE}

La Mission franco-syrienne pour l'étude de la citadelle de Damas ${ }^{1}$, nous a chargé des recherches d'archéologie du bâti monumental principalement dans le quartier de la salle à colonnes et dans le bâtiment sud-ouest, traditionnellement dénommé "palais», bien que ce rôle ne soit pas défini (fig. 1). Celui-ci comprend deux niveaux, tous deux antérieurs aux murs et aux tours sud et ouest de l'enceinte extérieure de la citadelle de fondation ayyoubide. Il est encore en cours d'étude archéologique et nous ne pouvons préciser sa position chronologique au-delà du fait qu'il est vraisemblablement très précoce dans le système défensif ayyoubide, voire un peu antérieur à celui-ci. Pour le quartier de la salle à colonnes, nous renvoyons le lecteur à la présentation synthétique proposée par A. Hartmann-Virnich dans son étude des portes nord et sud de la citadelle qui constitue une application monographique de la méthode exposée ici ( $c f$. infra).

Le fonctionnement technico-économique de ces chantiers médiévaux de construction est au centre de ces études en cours dont l'un des objectifs est de préparer les travaux de restauration et de mise en valeur du monument ${ }^{2}$. Au-delà d'une première présentation des recherches, récemment parues (BESSAC 2002; BoQVIST 2002; HaRTMANN-VIRNICH 2002), nous proposons ici un nouvel état de la question associant problématique, méthodologie et résultats. Mais ce travail est surtout un essai méthodologique qui s'inspire des études techniques sur la construction des fortifications antiques (BESSAC 1980; 1986; 1987; 1988; 1997) et qui aborde les questions souvent négligées dans ce secteur de la recherche. Nous soulignerons donc surtout ce point. Les résultats déjà obtenus offrent des aspects innovants, en particulier dans le domaine de l'organisation des chantiers de construction; nous les citerons donc, à titre d'exemple, pour illustrer la méthode. Les recherches de parallèles ne seront proposées qu'à partir de données issues des quelques études conduites dans le même esprit, dans la région ou ailleurs. Peu de manuels d'archéologie monumentale abordent l'architecture médiévale du Proche-Orient. Un esprit de recherche lexicographique, typologique et

\footnotetext{
${ }^{1}$ Dirigée par Sophie Berthier pour l'Institut Français d'Études Arabes de Damas (IFEAD) et par Edmond Al Ajji pour la Direction Générale des Antiquités et Monuments de Syrie (DGAMS). Soulignons ici toute l'aide que nous offre la partie syrienne grâce au concours de Sawsan Khalifeh et de Abir Haddad (architectes DGAMS), Ahmad El Bouch et Atef Abu Arraj (topographes DGAMS). Nous n'oublions pas l'importante contribution des architectes recrutés par la partie française de la mission: Jean Yasmine, Maria Saadeh et Bissane Sharif, que tous soient remerciés ici. 2 J.-C. Bessac, (UMR 154 du CNRS) coordonne en outre l'ensemble des études en cours sur le bâti de la citadelle et dirige les recherches de M. Boqvist (doctorante à l'Université de Paris IV) sur ce monument.
} 


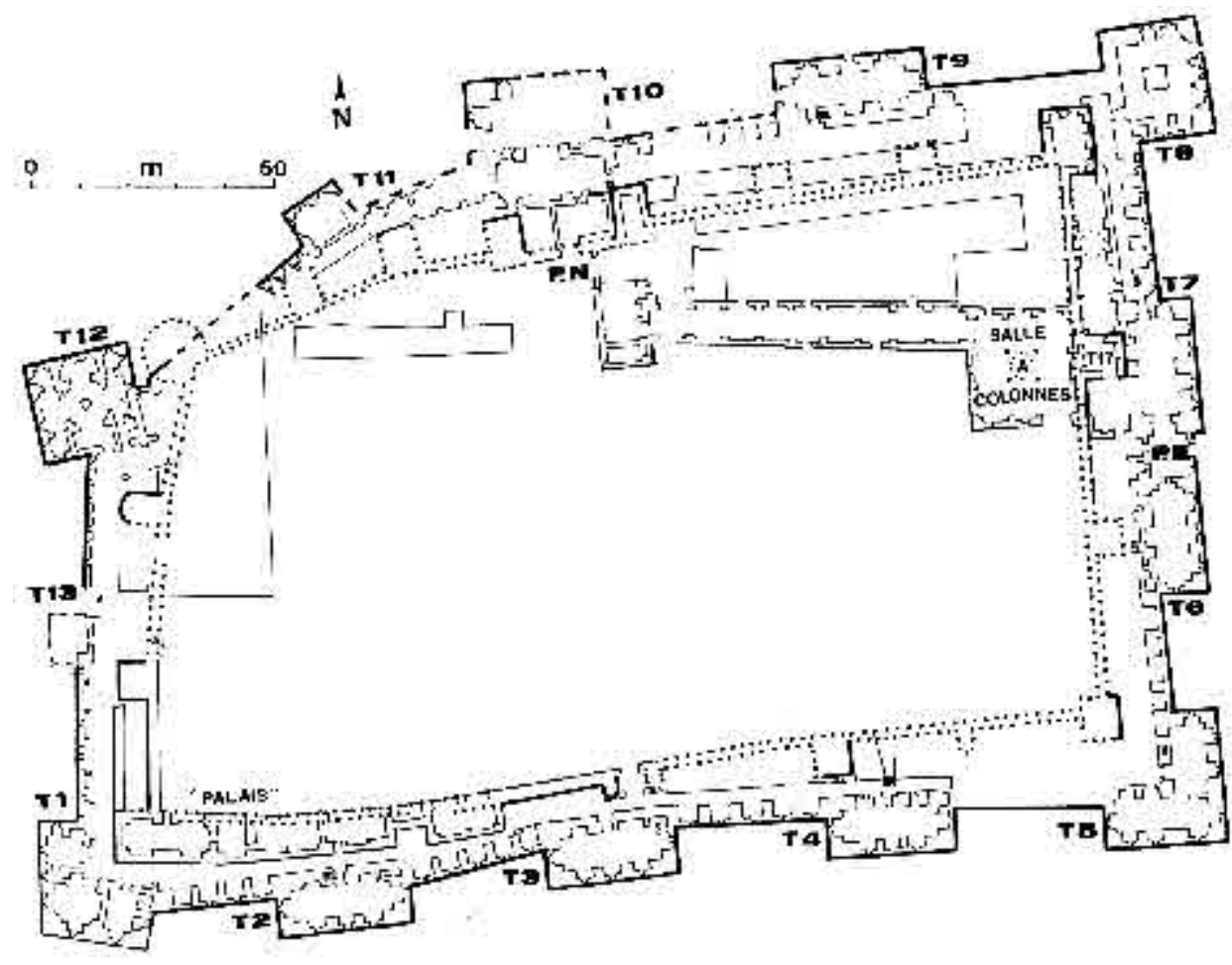

Fig. 1. Plan simplifié de la Citadelle de Damas situant ses principaux bâtiments (schéma établi d'après les documents originaux de la DGAMS) technique est donc indispensable ici. L'analyse de la construction repose sur trois grandes formes d'approches:

- un inventaire analytique complet de toutes les composantes architecturales sous la forme d'un catalogue général;

- une analyse technique thématique globale de la construction appuyée par des comparaisons;

—une synthèse archéologique des résultats qui doit répondre aux questions soulevées dans la perspective de la problématique générale du site.

\section{L'INVENTAIRE ANALYTIQUE DES STRUCTURES DE LA CITADELLE}

Même si les impératifs pratiques imposent parfois une autre marche, la progression géographique d'approche des grandes constructions de la citadelle est la suivante: côté sud et "palais», mur oriental, salle à colonnes et ses annexes, mur nord, côté ouest pour finir. Ces vastes ensembles sont ensuite découpés en grandes unités fonctionnelles (tours, courtines, bâtiments accolés) qui sont examinées dans un même sens de lecture, en soulignant leurs interrelations sous divers aspects (techniques, économiques, chronologiques, stylistiques). Chacune de ces unités est divisée à son tour en composantes essentielles, des plus vastes aux plus réduites (étages, salles, annexes), et, toujours lue dans un même sens selon la logique de construction, soit de bas en haut. Cette hiérarchie d'analyse est en outre adaptée à l'échelle des structures et très étroitement liée aux principes généraux d'avancement pratique de la construction. Par exemple, les chambres de tir et les meurtrières sont décrites en les situant par rapport aux composantes qui les enveloppent et à celles qui les constituent (voûtes, arcs, linteaux, voussoirs, piédroits). Chaque unité, sous-unité, composante et souscomposante est elle-même analysée dans le cadre de rubriques techniques qui sont spécialement adaptées à l'ouvrage. Les problématiques, les méthodes, le vocabulaire privilégiés ici sont ceux de l'archéologie de la construction traditionnelle en pierre.

\section{LES RUBRIQUES TECHNIQUES COMMUNES DE L'ANALYSE}

\subsection{Les dimensions}

Outre les données des plans, l'analyse des dimensions intervient dans les secteurs suivants:

- le calcul des surfaces et des volumes des salles et surtout des structures construites;

-les recherches métrologiques et modulaires sur les appareils des murs;

- la caractérisation dimensionnelle de familles de composantes architecturales (portes, arcs, piliers, etc.);

- les recherches sur les particularités géométriques des composantes architecturales, en particulier pour les couvrements. 


\subsection{Les matériaux et les matières premières de la construction}

Les matériaux de construction et les matières premières des chantiers de la citadelle sont étudiés sous l'aspect de leurs relations et interrelations avec les ressources naturelles locales mais aussi avec la définition de la commande de chaque partie du monument. Ils sont traités d'abord d'une manière générale, puis détaillés, si nécessaire, par composante architecturale, en soulignant leurs particularités et leur spécificité d'emploi. L'ensemble des matériaux est inventorié, mais leur description se limite ici à l'examen macroscopique. L'approche microscopique sera confiée à des spécialistes après définition de la problématique de cet examen par les archéologues.

À Damas, les calcaires durs et très durs prédominent et les bois d'œuvre sont très rares. Ces données de base sont fondamentales pour l'orientation de l'étude et pour l'interprétation du fonctionnement de ces chantiers. Par exemple, il est naturel que les composantes les plus ouvragées soient réalisées dans la pierre la moins difficile à travailler.

\subsection{Les appareils, les structures et le traitement des blocs et des joints}

Les choix techniques dans ce domaine sont surtout subordonnés à la nature des pierres. La question des appareils et des structures construites n'est donc abordée qu'après avoir identifié les diverses roches des murs et étudié leur aspect lithostratigraphique. Des essais de normalisation des hauteurs d'assise, entre 56 et $57 \mathrm{~cm}$, ont été tentés par les constructeurs de la salle à colonnes, dans le bas des murs. Mais c'est avant tout l'épaisseur naturelle des strates des calcaires locaux et leur fracturation géologique qui déterminent les modules des blocs. La suggestion d'une probable hauteur d'assise ayyoubide, entre 50 et $62 \mathrm{~cm}$, ne peut donc être mieux précisée (BOQVIST 2002).

Les bossages rustiques des façades intra-muros et extra-muros de la citadelle — pratique antique qui réapparaît alors dans la région - sont des bossages d'économie laissés bruts d'ébauche, ou de débitage, voire en l'état naturel, à cause de la grande résistance de ces pierres (fig. 2). L'analyse de ce type de bossages dans les ouvrages antiques invalidait déjà l'hypothèse d'un façonnage esthétique ou d'un dispositif destiné à dévier les projectiles (BESSAC 1995: 397-398). Les joints rubanés —également d'origine romaine- sont généralisés dans la citadelle et semblent cacher les irrégularités des contours de ses pierres, très difficiles à tailler, plutôt que d'obéir à une mode strictement esthétique.

\subsection{Les traces d'outils sur les pierres et leur interprétation}

Les traces d'outils et les caractérisations des tailles découlent aussi en partie de la nature de la pierre, mais leur prise en compte déborde très largement les aspects strictement techniques de l'extraction, du débitage et de la taille. L'impact de l'outil permet de l'identifier, de connaître ses dimensions et ses particularités, par exemple, pour le ciseau grain d'orge, sa largeur, le nombre et la forme de ses dents (BESSAC 1986a: 144-148). Les traces de cet outil sont d'ailleurs les plus communes, après celles de la broche, dans le quartier de la salle à colonnes (BOQVIST 2002) mais sont quasi absentes du "palais», mis à part dans son angle infé-

Fig. 2. Bossages d'économie présentés bruts d'extraction ou de débitage sur des blocs de calcaire local très dur dont le parement est cerné de joints rubanés qui masquent les irrégularités des arêtes. Tour 2, face sud (ph. J.-C. Bessac, CNRS)

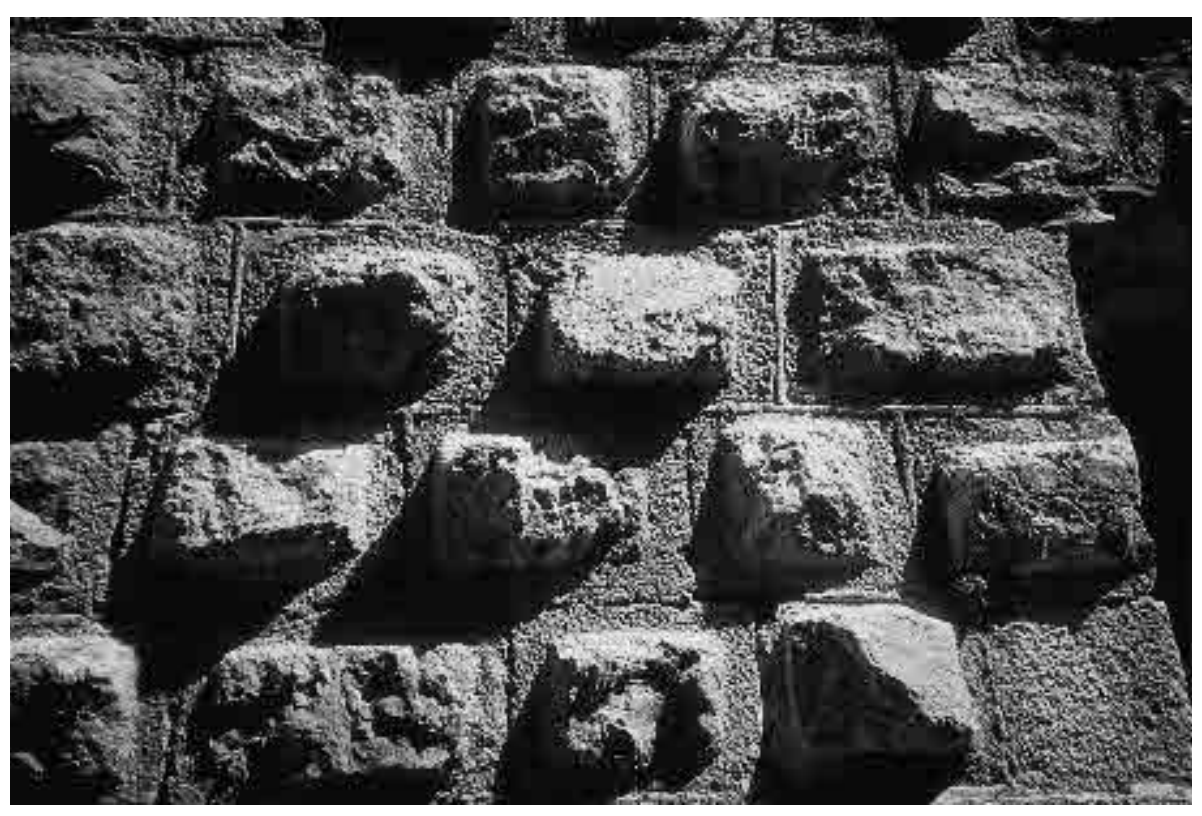




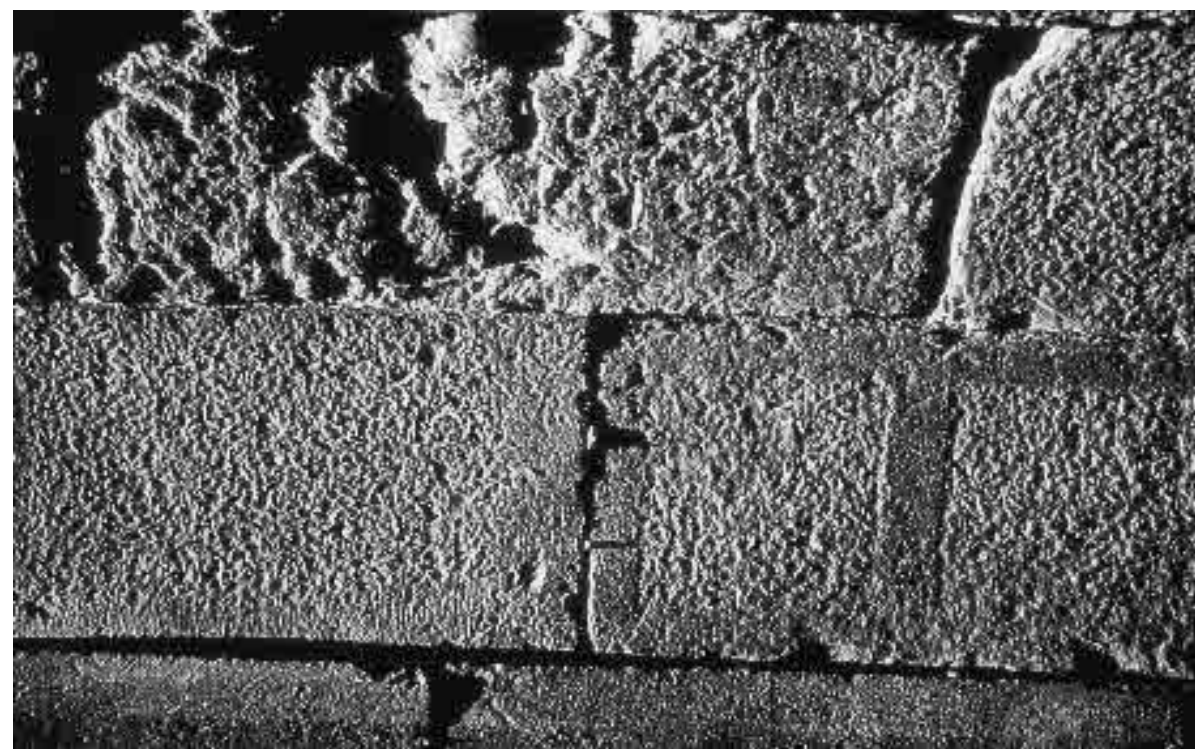

Fig. 3. Taille ornementale pointée fine cernée de marges régulières ciselées grain d'orge simulant un joint montant sur la retombée de la voûte rampante de l'escalier de la tour 10 (ph. J.-C. Bessac, CNRS) rieur nord-ouest. C'est le seul outil denté d'origine antique, et surtout romaine et byzantine en Syrie, qui s'impose à nouveau pour les ciselures des parements les plus soignés de la citadelle (fig. 3). Le marteau grain d'orge à tranchants perpendiculaires au manche, ou châhût $\hat{t}$, a peut-être été utilisé de manière très exceptionnelle pour la porte orientale. Les traces du ciseau droit sont rarissimes; l'extraction et le débitage n'ont laissé que quelques traces de coin de fer.

L'étude de la répartition, de la position, de la densité et de la variété des traces d'outil débouche, en outre, sur des informations techniques mais aussi anthropologiques, que nous limitons ici à quelques exemples.

\section{La distinction entre taille fonctionnelle et ornementale}

Les tailles pointées à la broche ont souvent un aspect différent selon qu'elles sont pratiquées sur des parements ordinaires ou sur des encadrements d'ouverture et des retombées de voûte, parties complexes et très visibles (fig. 3).

\section{La position de travail des constructeurs, à pied d'œuvre ou en ouvre}

La distribution des impacts sur les fonds de réceptacle des vantaux de porte est relativement homogène par rapport aux tailles des parements communs; cet aménagement était donc probablement taillé, ou pour le moins rectifié en place par un seul ouvrier.

\section{Le processus de la taille des blocs ou des pièces spéciales}

La disposition de certains coups d'outils révèle que la taille des éléments délicats, tels les couvrements de meurtrières, a nécessité un ajustage à sec par des spécialistes, sur les chantiers de taille de la citadelle; le même constat a été fait ailleurs (BESSAC, YASMINE 2002: 279).

\section{L'expérience des artisans}

Outre la précision et la netteté des faces et des arêtes des blocs, la maîtrise ou l'incompétence d'un tailleur de pierre se lisent souvent dans la répartition et la régularité de ses impacts d'outils. Les différences de niveaux professionnels sont nettement en faveur des spécialistes des ouvertures et surtout des décors (muqarnas, culots).

\section{La détermination du nombre d'intervenants}

La diversité des tailles de la porte ouest de la salle à colonnes révèle une certaine personnalisation des tailles et permet d'estimer le nombre des spécialistes qui l'ont réalisé entre quatre et sept, compte tenu des incertitudes dues aux variantes techniques individuelles. À titre de comparaison, les arêtiers de la voûte à pénétration du bastion oriental du château de Beaufort ont été confectionnés par quatre tailleurs de pierre qui ont personnalisé la finition des parements (BESSAC, YASMINE 2002: 296-297).

\section{L'exécution sommaire d'une tâche ou son affinage}

L'analyse des traces d'outils démontre que ces deux situations coexistent, notamment au sein de chacun des divers encadrements d'ouvertures du quartier de la salle à colonnes dont les parements sont très inégalement décorés. Mais les différences constatées dans ce domaine pour les meurtrières sont plus techniques qu'esthétiques. Les directives données aux ouvriers ne concernaient donc probablement 
pas l'apparence des parements, leur laissant ainsi une marge de liberté esthétique personnelle commune à d'autres lieux et périodes (BESSAC 1993: 169).

\subsection{Tailles fonctionnelles et ornementales: standardisation des aspects}

Notre vocabulaire et nos définitions des tailles médiévales du Proche-Orient se réferent surtout aux traditions occidentales et aux normes françaises du milieu du XXe s. qui les classent par ordre de rugosité décroissante (LE MAUSOLÉE 1976: 17-18). Ces référentiels sont cependant trop normatifs; ils ont donc été adaptés et complétés grâce aux études déjà conduites ou en cours sur divers sites antiques et médiévaux de la péninsule Arabique: Pétra et Jérash, (Jordanie), Hégra (Arabie Saoudite), Maïn, Shabwa et Marîb (Yémen), Suweida, Bosra, Doura-Europos, Halabiyya-Zenobia (Syrie) et Beaufort au Liban (BESSAC 1997: 91-98; id., 1998a: 173-230; id. 1998b: 231-282; BESSAC, BRETON 2002; BeSSAC, YASMINE 2002).

Outre la dénomination de la taille, le comptage des impacts par $\mathrm{dm}^{2}$ définit précisément la rusticité ou la finesse du traitement des parements. S'il s'agit d'impacts directionnels, tels ceux des tailles smillées ou ciselées grain d'orge, respectivement réalisées avec une broche (ou un pic) et un ciseau grain d'orge (BESSAC 1986: 20 et 146), la mention de l'orientation des impacts, oblique, croisée, perpendiculaire aux arêtes ou quelconque, apporte des précisions complémentaires, notamment sur une éventuelle standardisation de la taille. L'étude de la citadelle, en dehors de ses parties décorées (muqarnas, mihrab, culots) n'a révélé qu'un témoignage de standardisation stricte de la taille dans l'angle nord-ouest de la façade du rez-de-chaussée $\mathrm{du}$ "palais»: elle est ciselée grain d'orge perpendiculaire (fig. 4); le reste est traité de manière fonctionnelle et assez disparate.

Ces traitements incluent aussi l'aspect brut d'extraction ou de débitage et l'état naturel, qui varient selon le type de roche et la nature de la face géologique: diaclase, joint de stratification, etc., (fig. 2). Dans les productions les plus affinées, ils comprennent des tailles décoratives composites qui associent, ou juxtaposent parfois plusieurs traitements élémentaires. Tel est le cas de la taille pointée fine cernée d'une marge régulière ciselée grain d'orge (fig. 3) dont le principe est d'origine antique, bien qu'elle soit issue de la péninsule Arabique (BESSAC 1998a: 189-190). Elle se trouve souvent sur les encadrements de porte de la citadelle ou à la naissance de ses arcs et voûtes (BOQVIST 2002: 94; HartManN-Virnich 2002: fig. 3 et 6 ; $i d$. infra). La répartition des impacts sur chaque partie du parement et l'éventuelle délimitation des traitements sont précisées (pointillés d'impacts de broche ou ligne incisée au ciseau grain d'orge).

La forme du relief éventuel des parements est prise en compte en plus de la taille de son épiderme; la combinaison des deux constitue souvent un traitement spécifique comme les bossages décoratifs. Le plus connu est traité en table, mais il est plutôt propre aux monuments antiques et croisés. Le travail de tradition franque est rarissime dans la citadelle mais les remplois d'éléments romains nécessitent un élargissement typologique aux productions antiques. Un autre type de bossage ornemental plus isolé, la pointe de diamant, est présent dans quelques angles saillants, côtés

Fig. 4. Taille standard ciselée grain d'orge perpendiculaire aux arêtes des blocs. Angle nord-ouest de la façade septentrionale du bâtiment sud-ouest («palais») au rez-de-chaussée (ph. J.-C. Bessac, CNRS)

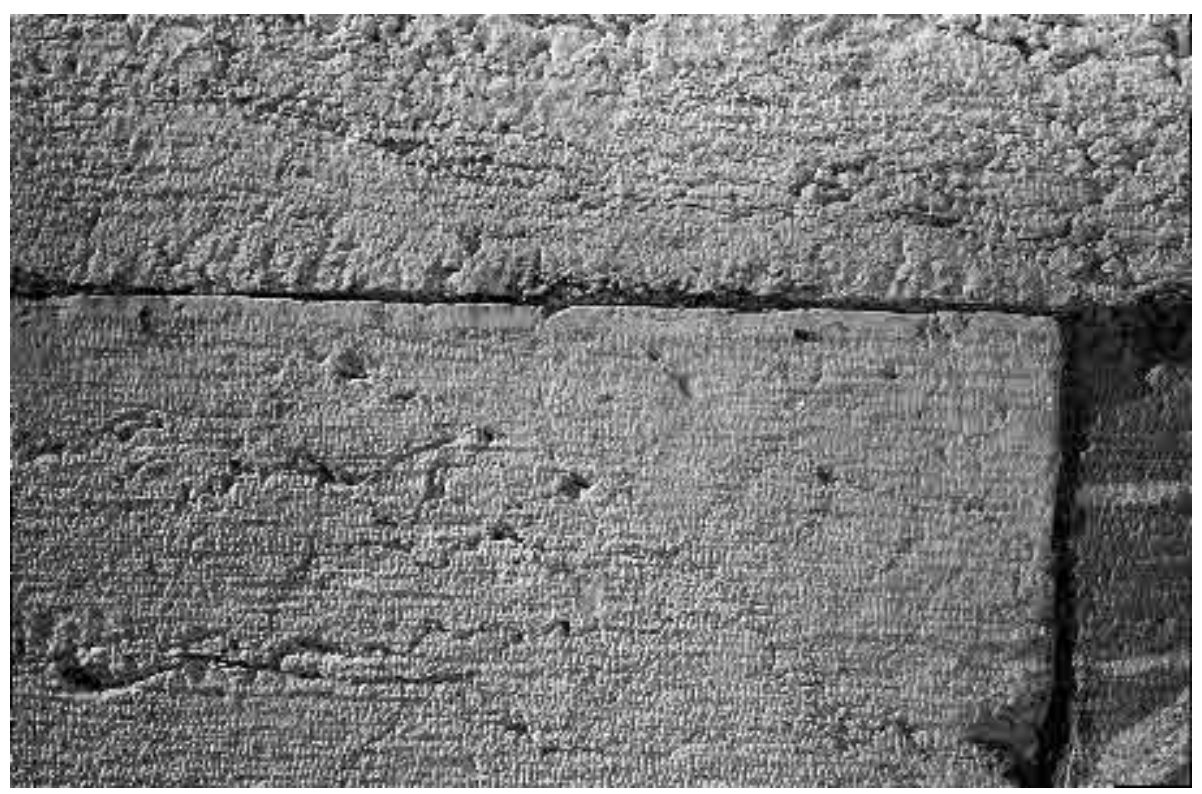


sud et est (fig. 5). Des exemplaires plus nombreux, et probablement plus anciens, existent dans la région à Beaufort (BESSAC, YASMINE 2002: 272, fig. 36).

\section{LES RUBRIQUES STRUCTURELLES DES COMPOSANTES}

\subsection{Les arcs}

La citadelle de Damas comporte de nombreux types d'arcs qui seront présentés avec les couvrements. Trois de leurs grandes spécificités sont traitées ici.

\section{Les types d'assemblages}

Leur forme propre est précisée, tels les joints en coupe, à crossettes, à tenons, etc. L'assemblage apparent le plus étonnant est celui de l'arc trilobé de la porte nord qui comporte des voussoirs inversés, le côté large du prisme placé vers le bas (HARTMANN-VIRNICH 2002: id. infra). Le principe caché de la coupe interne de leurs joints reste à découvrir car la résistance du mortier est insuffisante pour expliquer leur stabilité. Une plate-bande en fausse coupe pose le même problème dans la tour 9.

\section{Leur composition et leur disposition}

La présence et la disposition de sommiers, contre-sommiers, clé - lorsqu' elle existe-, contre-clé, et le nombre de claveaux sont précisés. Par exemple, la composition de plusieurs arcs des chambres de tir et des meurtrières de la tour 7 differe suggérant un travail de diverses équipes.

\section{Le traitement des extrados}

Il peut être concentrique, en escalier, mixte, irrégulier, etc. C'est aussi un indice complémentaire pour définir les pratiques propres à chaque équipe.

\subsection{Les voûtes}

La même stratégie d'étude s'applique aussi aux voûtes, mais leur typologie varie un peu, notamment pour les voûtes d'arêtes et les coupoles. Il existe aussi des exemplaires maçonnés ou mixtes, en pierre de taille, pour leurs retombées et leurs formerets et en pierre de tout-venant pour le reste. Il importe donc de les caractériser ainsi que leur mode de raccord entre pierre taillée et maçonnerie. Par exemple, les retombées en pierre de taille des voûtes du quartier de la salle à colonnes se distinguent de celles du "palais» par leur coupe et leur composition; il en est de même pour la nature et la disposition de leurs pierres de tout-venant maçonnées au-dessus.

Les typologies actuelles des couvrements sont insuffisantes, il a donc fallu pallier leurs lacunes pour inclure tous les cas de figure. Parmi les particularités des arcs et des voûtes, leurs défauts d'exécution sont très attentivement analysés.

\subsection{Les liaisons et les raccords de la construction}

Des murs ou des composantes qui se joignent sans liaison sont souvent considérés, a priori, comme résultant d'ajouts ou de modifications. Cette règle souffre des exceptions que l'on identifie en observant très méticuleusement les raccords. A contrario, des liaisons de mur, apparemment correctes, cachent parfois des phases différentes de construction. Les liaisons et les raccords se divisent en deux grands groupes.

\section{Les liaisons et les raccords des structures maîtresses}

Leur identification repose sur des indices d'antériorité ou de postériorité d'une construction par rapport à l'autre. Cela concerne, notamment: l'oblitération partielle ou totale d'une taille ornementale, d'un enduit, ou de mortier de

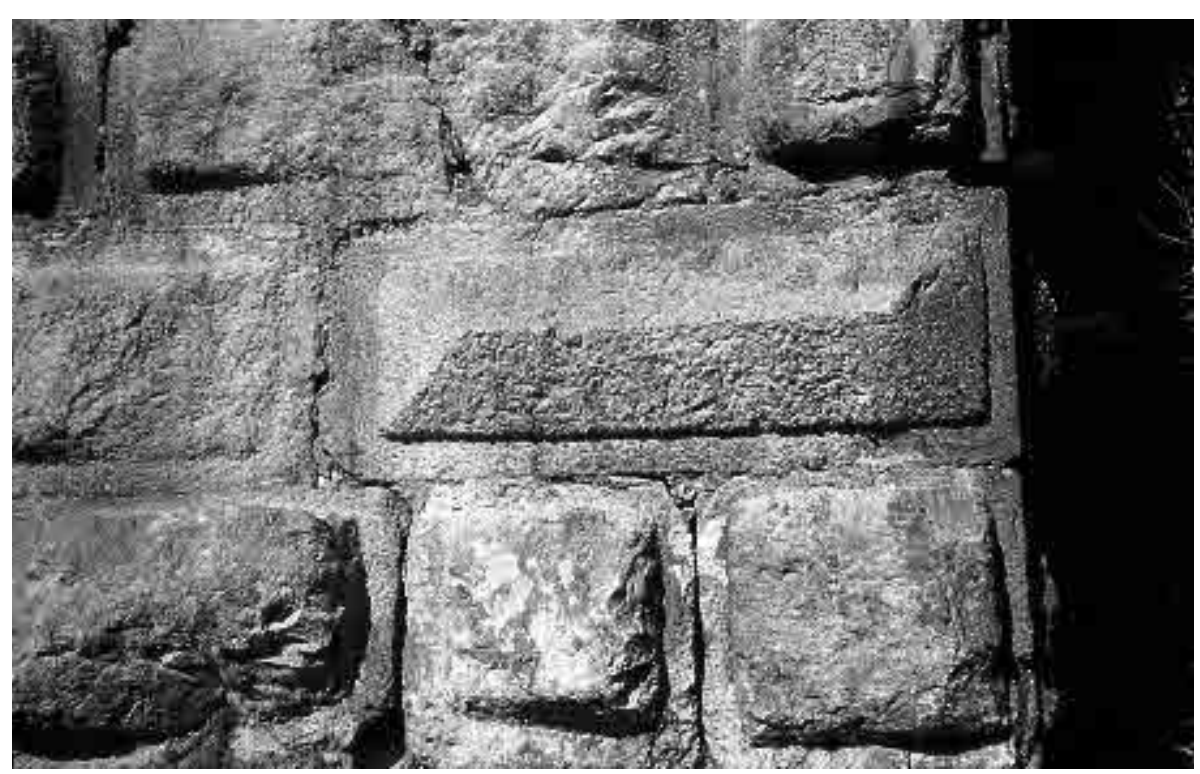

Fig. 5. Bossage ornemental en pointe de diamant à l'angle nord-est de la tour 7 (ph. J.-C. Bessac, CNRS) 
jointoiement sur un mur sur lequel une autre structure prend appui. Tel est le cas de la façade extérieure occidentale de la salle à colonnes qui est plus ancienne côté tour 17 qu'à l'intérieur, bien qu'elle appartienne au même mur.

Mis à part la découverte exceptionnelle d'objets archéologiques bien situés dans le temps (céramiques, monnaies, etc.), la datation absolue de murs se fonde parfois sur des techniques de construction caractéristiques d'une période: matériaux, outils, procédés de taille ou de mise en œuvre, appareils employés seulement à une époque donnée. C'est ainsi que les murs seldjoukides de la citadelle se distinguent des exemplaires ayyoubides — d'habitude en grand appareil bien taillé- soit par l'usage du petit appareil, soit par l'emploi de grands blocs à peine équarris, souvent à partir de récupérations antiques. Il reste à obtenir des repères typologiques sûrs pour établir des chronologies plus fines, en particulier entre les constructions de la deuxième moitié du XII ${ }^{e}$ s. et de la première moitié du XIII ${ }^{e} s$.

\section{Les liaisons et les raccords entre composantes d'un même ensemble}

Les anomalies de liaisons ou de raccords entre les composantes d'un même ensemble, notamment entre les murs et la porte d'une salle, ou entre deux parties d'une ouverture, sont trop souvent interprétées comme des changements dans l'édifice original. Cette conception moderne se révèle de plus en plus discutable pour les œuvres médiévales et antiques. L'autonomie de construction de composantes d'un même bâtiment est connue dans les fortifications antiques (BESSAC 1997: II, 140-147) et dans la construction romane occidentale (BESSAC, PÉCOURT 1995: 110-116). L'analyse est donc pratiquée sans a priori. Les moindres irrégularités de liaisons entre les diverses parties d'un grand ensemble sont notées en plus des anomalies internes à la composante en cours d'analyse. Toutes les particularités techniques de la construction sont étudiées de chaque côté des liaisons pour mettre en évidence leurs différences et leurs analogies. Le découpage d'un chantier en petites tranches de construction réparties entre diverses équipes de spécialistes ne se distingue souvent des reprises du bâti de dates différentes que par des indices très réduits.

La porte orientale de la salle à colonnes, qui comprend une face traitée avec une certaine ostentation, côté est, et l'autre plus sobrement, illustre bien cette situation. Ses pierres présentent des hauteurs d'assise différentes de chaque côté et se raccordent par l'intermédiaire de décrochements dans l'embrasure. Il pourrait s'agir là du résultat de deux phases de construction si l'analyse ne démontrait pas l'impossibilité de mettre en place chacune de ces deux parties de la porte de façon autonome. La complexité de l'ouvrage étant très différente sur ses deux faces, ces encadrements ont été certainement taillés indépendamment par deux équipes distinctes de spécialistes, mais la totalité de la porte a été posée dans la même étape de construction. D'autres interventions de spécialistes ont été identifiées dans la citadelle, en particulier dans sa porte orientale en cours d'étude par A. Hartmann-Virnich ( $c f$. infra). Mais cette division du travail semble aussi de règle entre des composantes moins prestigieuses, telles les meurtrières et les chambres de tir.

\section{LES MARQUES LAPIDAIRES}

Les marques lapidaires ont été prises en considération jusqu'ici au Proche-Orient essentiellement pour les réalisations franques. Il existe pourtant des marques lapidaires propres aux constructions de tradition islamique, notamment dans la citadelle, mais ici les exemplaires francs sont rarissimes. Nous traitons ce sujet dans une perspective d'étude technique et anthropologique des chantiers de construction plutôt que dans une optique d'épigraphiste, selon les nouvelles tendances qui se dégagent en matière de glyptographie (Dopéré 2000: 191-224; id. 1997: 153-198; KILL 1997: 249-182). Les quelques marques franques observées dans la tour 2 posent un problème historique majeur: s'agit-il de prisonniers ou bien d'équipes de tâcherons qui se louaient aussi bien d'un côté que de l'autre? La question vaut la peine d'être posée. Les différences techniques entre ces deux catégories de marques nécessitent des méthodes de relevé et d'inventaire adaptées à chacune.

\subsection{Les marques de tradition islamique}

Il semble exister deux sortes de marques de tradition islamique qui ne se distinguent que par leur fonction. Elles sont toutes deux très sommairement incisées à la broche surtout sur des bossages rustiques, ce qui complique souvent leur identification (fig. 6). Elles transparaissent parfois à l'état résiduel sur des parements aplanis. Leurs dimensions varient de quelques centimètres à toute la longueur d'un parement. Leur position sur celui-ci n'obéit pas à une règle bien qu'elles se trouvent plutôt vers son milieu.

Les marques sont relevées grandeur nature, puis réduites au $1 / 10^{\mathrm{e}}$. Faute de pouvoir les reporter dès maintenant sur un dessin pierre à pierre pour les situer dans l'édifice, nous utilisons provisoirement le système de repérage et de marquage de la construction traditionnelle. Il consiste à identifier le mur, l'assise et le bloc par des numéros d'ordre progressant de bas en haut et de gauche à droite (NOËL 1968: 75; ALADENISE 1982: 42-47). 


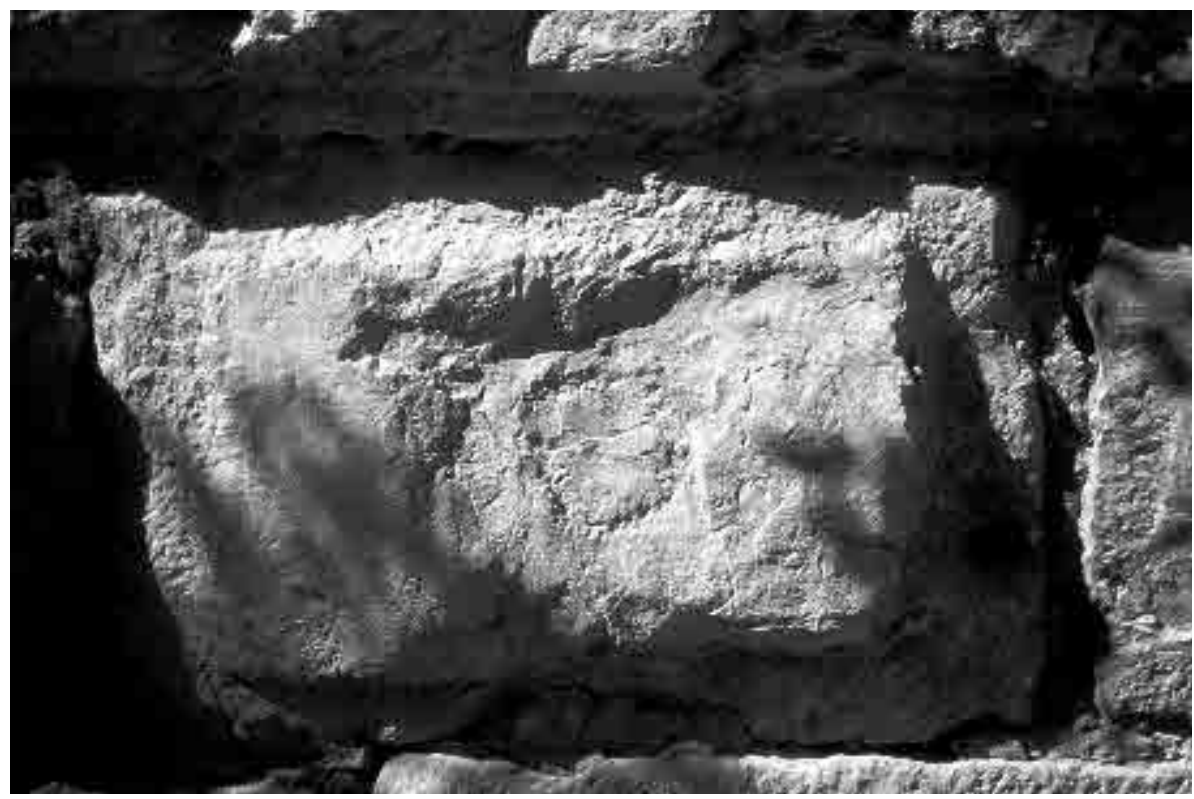

Fig. 6. Marque lapidaire médiévale islamique ronde sur un bossage d'économie brut de débitage. Façade orientale de la tour 7 (ph. J.-C. Bessac, CNRS)
La fonction de ces marques n'a pas été encore définie sûrement. Toutes se trouvent sur des bossages rustiques et certaines d'entre elles sont sectionnées par la ciselure périmétrale réalisée au début de la taille: elles ont donc été certainement incisées en carrière. L'étude en cours de leur répartition dans le quart nord-est de la citadelle révèle aussi que les exemplaires en ligne brisée se trouvent presque uniquement sur les courtines (fig. 7), alors que les spécimens ronds sont réservés aux tours (fig. 6). L'hypothèse d'une identification entre les livraisons de lots de pierres destinés aux courtines et aux tours est donc envisageable. La différenciation entre les deux commandes pourrait alors s'expliquer par une plus grande exigence de qualité des blocs qui sont destinés aux tours dont les angles, les arcs, les voûtes et les ouvertures nécessitant une taille assez complexe et soignée. Les quelques marques identifiées dans la salle à colonne sont également rondes et semblent renforcer cette hypothèse. Toutefois, elles se trouvent surtout sur des blocs particuliers: support de base de colonne et seuil. Elles pourraient donc signaler des blocs hors normes comme nous l'avons remarqué dans les murs hellénistiques de Doura-Europos (BESSAC 1997: 180-181). Une telle fonction de ces marques a été identifiée dans le château de Beaufort; mais là, ces signes lapidaires sont un peu plus diversifiés et une incision assez soignée a été possible car ils se trouvent presque tous sur des parements bien aplanis à la broche (BESSAC, YASMINE 2002: 308, fig. 88-90).

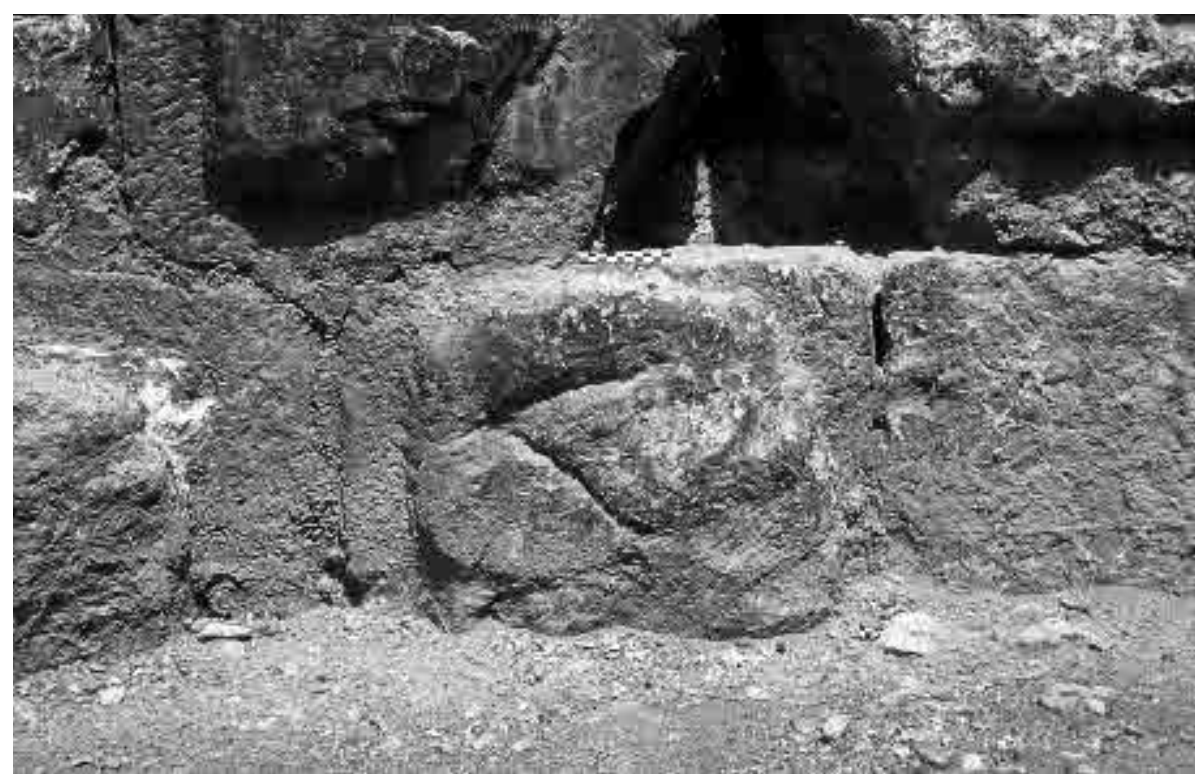

Fig. 7. Bossage d'économie marqué d'une ligne brisée. Façade orientale de la courtine initiale, au sud du «palais», à l'emplacement d'une reprise (ph. J.-C. Bessac, CNRS) 


\subsection{Les marques lapidaires de tradition franque}

Les mêmes principes généraux d'inventaire et de relevé sont également applicables ici, mais il faut procéder avec plus de précision vue l'étroitesse du sillon de gravure de ces marques constituées de lettres latines (fig. 8). Connues ici uniquement à l'intérieur des bâtiments, elles se distinguent aussi des précédentes par leur support qui est constitué de parements plans et unis. Les données incluent donc, outre la forme de la lettre, sa technique d'incision et le type de taille du parement. Cette catégorie de marque autorise aussi une recherche du coup de main des scripteurs en vue de les caractériser et de les situer par rapport à l'exécution de la taille. L'hypothèse de marques individuelles de tailleurs de pierre spécialisés dans la production d'arcs et de voûtes paraît plausible. Mais aucun indice ne permet de parler de marques de tâcherons, le mode de paiement et le statut de ces spécialistes étant inconnus. Sans écarter cette éventualité, ces marques pourraient correspondre à une identification des tailleurs à des fins de contrôle de la qualité de leur production individuelle. Il est possible d'envisager aussi une comptabilité de ces blocs en vue du paiement de l'employeur ou du maître des tailleurs de pierre.

\section{LES REMPLOIS}

L'étude des remplois constitue une rubrique à part entière qui vise deux objectifs: la connaissance du mur médiéval et l'identification des monuments antérieurs d'où proviennent ces blocs.

\subsection{Les données propres à l'opération du remploi}

Le bloc de remploi est d'abord caractérisé dans sa nouvelle fonction, puis situé précisément dans le mur, selon les règles adoptées pour les marques lapidaires. Il faut aussi déterminer la part de retaille, par rapport à l'élément originel, et indiquer la nature initiale du bloc: linteau, piédroit, colonne, chapiteau, etc. La roche du remploi est identifiée et comparée aux variétés de pierre spécialement extraites pour la construction médiévale. C'est ainsi que l'on repère parfois des blocs de même nature mais qui sont trop retaillés pour reconnaître en eux des remplois. Tel est le cas de blocs antiques réutilisés dans les constructions postérieures aux réalisations seldjoukides, comme celui remployé dans la porte ouest de la salle à colonne (BOQVIST 2002: fig. 3).

\subsection{L'origine du remploi}

L'analyse des remplois, bien que comparable à celle des blocs médiévaux, s'en distingue par sa plus grande complexité qui découle des lacunes de ces vestiges et surtout de la présence de décors moulurés et sculptés. Elle concernent

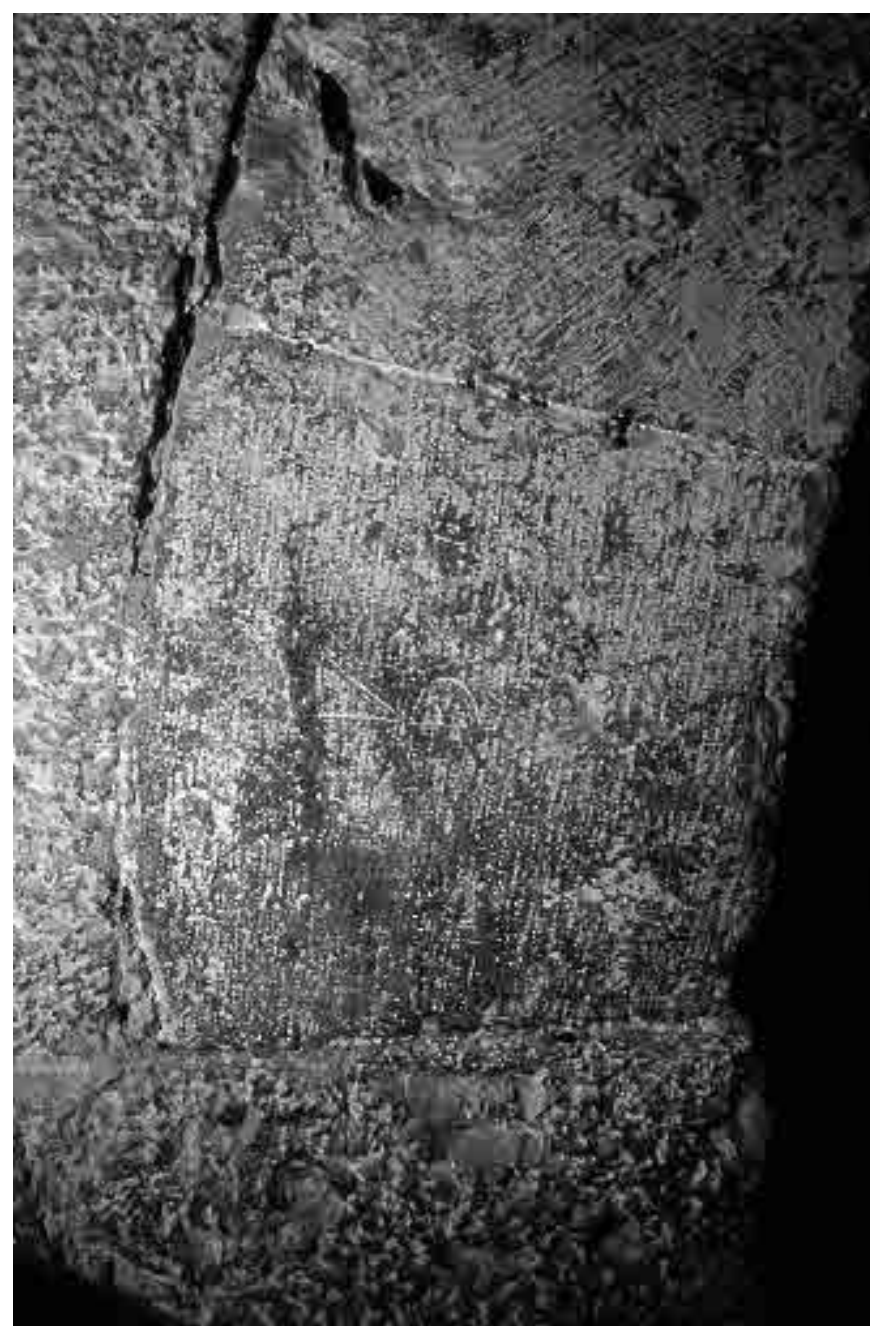

Fig. 8. Marque lapidaire de tradition franque sur la porte d'entrée de la tour 3 (ph. J.-C. Bessac, CNRS)

la métrologie, la forme et le décor. Les techniques de ces productions romaines sont beaucoup plus complexes que celle des ouvrages défensifs médiévaux et nécessitent l'intervention de spécialistes. Les éléments d'une porte monumentale romaine, ou d'un arc de triomphe ont été ainsi identifiés dans les remplois de la tour 7 de la citadelle (BESSAC 2002: fig. 7).

\section{L'ÉTUDE THÉMATIQUE DU BÂTI MONUMENTAL}

Les données de l'inventaire analytique permettent d'aborder la grande étape de l'étude thématique. Celle-ci regroupe les caractères de la construction par phase chronologique et pose les jalons d'une typologie chronologique des techniques de construction. Cette synthèse est comparative à l'intérieur du monument mais aussi en dehors. La majorité des éléments du catalogue sont donc repris dans l'exposé thématique. Cette seconde phase de la recherche n'étant qu'amorcée, seuls quelques exemples sont présentés ici. 


\subsection{Les éléments généraux de la fortification}

La présentation d'une vue d'ensemble de l'ouvrage s'impose, comme dans l'inventaire, mais ici, les regroupements analogiques priment sur l'ordre géographique. Commencer par les plus grands éléments et les associer par catégories est donc impératif. Par exemple, dans la citadelle, il faut traiter à part les tours d'angle (n. ${ }^{\circ} 1,5,8$ et 12 ), les tours protégeant les grandes entrées (n. ${ }^{\circ}$ 6, 7, 10 et 13 et, en moindre mesure, la tour n. ${ }^{\circ} 3$ ) pour terminer par les tours communes (n.o $2,4,9$ et 11 ).

\subsection{L'étude thématique des éléments architecturaux}

Notre démarche méthodologique implique que les éléments architecturaux soient abordés et regroupés par ensembles de fonctions et d'échelles homogènes. Les grands thèmes de l'inventaire sont donc repris ici de façon autonome: ouvertures, couvrements, aménagements architecturaux divers, etc.

\section{Des ouvertures aux couvrements}

La typologie technique et chronologique des ouvertures dépend de leur fonction qui conditionne aussi leur construc- tion, leur mode de couvrement et leur système de fermeture. Les ouvertures d'une fortification se divisent en cinq grandes catégories: les portes principales, les portes secondaires et poternes s'ouvrant sur l'extérieur, les portes intérieures, les fenêtres et les meurtrières. Il est indispensable, d'un point de vue typologique, de les analyser avec leur couvrement. Mais il faut aussi reprendre ce dernier thème de manière intrinsèque et détaillée puisqu'il s'applique à une gamme beaucoup plus large de composantes architecturales.

\section{Une approche systématique des couvrements}

Les couvrements concernent les ouvertures mais aussi des salles, des couloirs, des escaliers; ils regroupent les arcs et les voûtes. La citadelle comprend au moins quinze grands types de couvrements:

—le linteau monolithe, considéré aussi comme un arc plat;

-la voûte plate, constituée d'une suite de linteaux monolithes;

-la voûte plate à décrochements, composée de linteaux monolithes décalés d'un degré dans les couvrements d'escaliers;

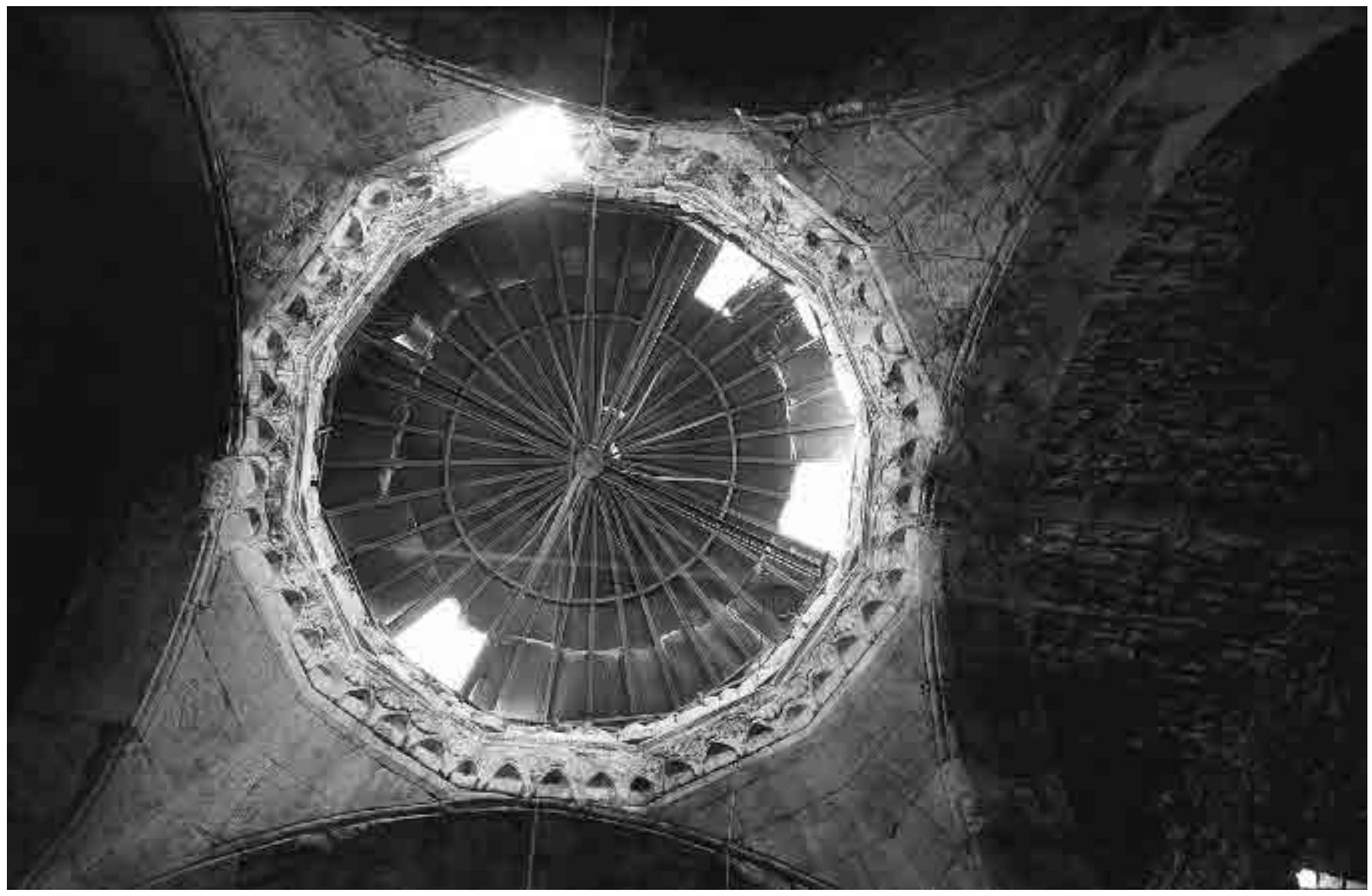

Fig. 9. Dôme tambour décoré de muqarnas constituant le couvrement de la travée centrale de la salle à colonnes (ph. J.-C. Bessac, CNRS) 
—la plate-bande appareillée, comprenant d'habitude une clé, deux sommiers et souvent plusieurs claveaux;

-l'arc brisé, le plus commun dans la citadelle;

—l'arc segmentaire, également très commun, mais avec une fonction réduite à la décharge des linteaux;

-l'arc outrepassé, exceptionnel et réservé à des ouvertures d'une certaine importance.

-la voûte segmentaire, susceptible de couvrir de grands espaces en étant bien contrebutée;

-l'arc polylobé, couvrement d'apparat que l'on ne trouve que dans la porte nord de la citadelle sous une forme trilobé;

—la voûte en berceau, généralement brisée à la citadelle;

-la voûte rampante, réservée aux couvrements de certains escaliers;

-la voûte d'arêtes, d'habitude maçonnée, sauf dans ses retombées et au-dessus des paliers et des repos d'escalier;

-la voûte en demi-coupole, qui n'existe ici qu'en taille réduite dans le mihrab près de la tour 7 et au-dessus de la porte orientale (HARTMANN-VIRNICH infra);

-la voûte en demi-arc-de-cloître, connue en deux exemplaires au-dessus de paliers d'escalier;

-le dôme à tambour, couvrant la salle à colonnes (fig. 9) et une salle au nord de la tour 17.

Chacun de ces couvrements peut être combiné avec d'autres ou inclure plusieurs sous-catégories. Tel est le cas de la plate-bande qui est, par exemple dans les petites portes du couloir à l'ouest de la salle à colonnes: à tenons avec des joints opposés en losange tronqué, à l'est, et en double queue d'aronde à l'ouest. Ces variantes sont déterminées par le tracé spécifique de leur courbe ou de leurs joints, voire les deux quelquefois.

L'analyse géométrique des arcs brisés prouve l'usage d'au moins sept formules différentes de tracé d'exécution (fig. 10). Elles sont souvent mêlées dans un même ensemble architectural sans modifier l'apparence générale du couvrement (BOQVIST 2002: fig. 8). Et c'est là l'un des points forts de cette recherche car il pose le problème de l'intervention simultanée d'équipes de constructeurs de traditions techniques différentes. Cette approche permet également d'aborder les connaissances et les pratiques géométriques des équipes de base du bâtiment, alors que jusqu'ici, seule la science des «maitres d'œuvre» a monopolisé la recherche.

\section{UN PREMIER BILAN DE LA RECHERCHE ET SES PERSPECTIVES}

L'exemple des tracés de couvrements, que l'on vient de voir, est certes l'un des plus intéressants. Il révèle que l'ampleur de la citadelle et son caractère défensif obli- geaient le regroupement, dans une courte période, d'un maximum de constructeurs, probablement recrutés en diverses régions du pays. Dans le seul domaine de la géométrie, la variété des compétences des équipes est prouvée par le tracé des ostentatoires muqarnas, comme ceux de la porte orientale et de la salle aux colonnes et des discrets décors des escaliers (tours 3 et 10). Mais cette spécialisation des équipes et leur qualification révélées par ces réalisations spécifiques et complexes, se concrétise aussi dans les appareillages ardus de certains éléments de voûtes, tels les sommiers de la première salle de la porte nord (HARTMANN-VIRNICH 2002: fig. 10; id. infra). Même s'il ne s'agit pas de stéréotomie, telle que la définissent les professionnels de la pierre (ALADENISE 1982: 162-163), cela reste des exercices de géométrie de haut niveau. La spécialisation des équipes suggère aussi une certaine préfabrication qui irait jusqu'à l'assemblage au sol des composantes élémentaires, comme les arcs ou les encadrements d'ouverture (portes et meurtrières). La mise en œuvre de ces pierres n'était peut-être pas confiée aux mêmes équipes qui les taillaient.

Les tailles ornementales ne sont pas normalisées, mais elles semblent suffisamment individualisées pour évoquer l'hypothèse d'une forme d'identification propre du travail de chaque tailleur de pierre dans les composantes architecturales soignées. Malgré la rareté générale des marques lapidaires, soulignons aussi l'intérêt de l'étude des deux variétés de signes. Alors que le groupe de tradition islamique parait identifier une spécificité qualitative des blocs au départ de la carrière, les marques franques pourraient signaler un travail individuel. Cette recherche devrait aussi apporter son lot d'informations innovantes, en particulier sur le fonctionnement des chantiers de construction mais aussi sur l'histoire de la citadelle.

Les rubriques de base: matériaux, outillage, appareils et structure, traces d'outils, tailles, liaisons, etc. prennent leur pleine ampleur lorsqu'elles sont considérées de ce point de vue technico-économique, mais aussi et surtout anthropologique. Cette approche révèle, dans la citadelle postérieure aux murs seldjoukides, un net regain de la rigueur et des pratiques monumentales gréco-romaines de la région. Celui-ci touche des domaines aussi divers que l'organisation du travail avec l'intervention d'équipes très spécialisées, les liaisons de structures, les applications géométriques, l'outillage, les tailles, les joints, l'usage de certaines marques lapidaires. La datation de ces évolutions de la construction reste à préciser. Compte tenu de la rusticité des murs seldjoukides de la citadelle — les seuls connus dans la proche région - il semble exister une franche coupure technique entre les constructions défensives de Syrie 

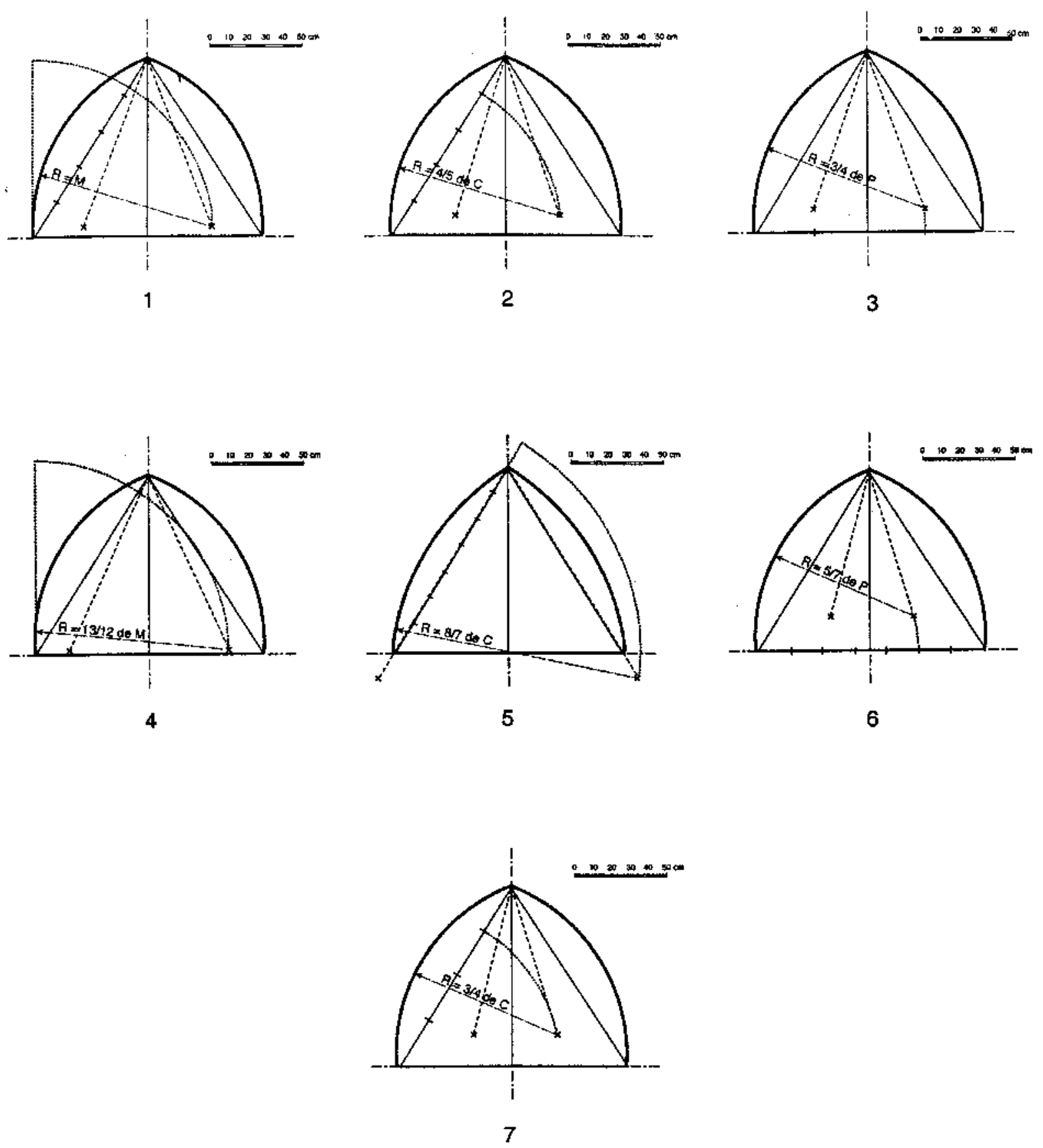

Fig. 10. Schéma montrant sept principes géométriques différents qui ont été utilisés pour obtenir des arcs brisés de la citadelle. Les rayons (R) et les centres sont déterminés et positionnés à partir du triangle inscrit dont la correspondance avec l'arc est la suivante: base = portée $(P)$, côté $=$ corde $(C)$, hauteur = montée $(M)$ (dessins M. Boqvist

de tradition gréco-romaine, voire byzantines, et les ouvrages postérieurs à cette enceinte initiale. Mais, en matière d'ouvrages militaires dans la région de Damas, il faut reconnaitre que nous ne savons presque rien sur la période antérieure à la deuxième moitié du XIIe $s$. C'est donc dans ce sens que nous souhaitons renforcer nos investigations sur le bâti monumental de la citadelle. Le programme est toutefois très vaste et il sera difficile de le réaliser en totalité dans les délais impartis à cette opération. Mais la présente phase d'étude débouche déjà sur des résultats décisifs pour la compréhension du fonctionnement de cette catégorie de grands chantiers médiévaux et le projet de conservation du monument pourra bénéficier de ces informations inédites. 


\section{Bibliographie}

Aladenise V., 1982, Technologie de la taille de pierre, Paris, Librairie du Compagnonnage.

BESSAC J.-C, 1980, Le rempart hellénistique de Saint-Blaise (Saint-Mîtreles-Remparts, B.-du-Rh.): Technique de construction, Documents d'Archéologie Méridionale, 3, pp. 137-157.

—, 1986a, L'outillage traditionnel du tailleur de pierre de l'Antiquité à nos jours, Paris, CNRS (Revue Archéologique de Narbonnaise, suppl. 14).

—, 1986b, Approche des problèmes posés par la construction des remparts grecs en pierre, Leriche P., TrÉZINY H. (éds.), La fortification dans l'histoire du monde grec, Paris, pp. 273-282.

—, 1987, Matériaux et construction de l'enceinte augustéenne de Nîmes, Les enceintes augustéennes dans l'Occident romain, Bulletin de l'Ecole Antique de Nîmes, 18, pp. 25-38.

—, 1988, Les procédés de construction des remparts de pierre de Doura-Europos: Questions de méthodologie, Syria, LXV, pp. 297-313.

—, 1993, Traces d'outils sur la pierre: méthodes d'étude et interprétation, FRANCOVICH R., (dir.) Archeologia delle attività estrattive e metallurgiche, Florence, pp. 143-176.

—, 1997 La construction des fortifications hellénistiques en pierre de Doura-Europos (Syrie), Thèse d'histoire, Université de Rennes, 3 vol.

—, 1998a, Techniques de construction, gravure et ornementation en pierre dans le Jawf (Yémen), BRETON J.-F. (éd.), Fouilles de Shabwa III: Architecture et techniques de construction, Beyrouth, IFAPO, Bibliothèque Archéologique et Historique, CLIV, pp. 173-230.

—, 1998b, Le travail de la pierre à Shabwa (Yémen), BRETON (J.-F.) (éd.) Fouilles de Shabwa III: Architecture et techniques de construction, Beyrouth, IFAPO, Bibliothèque Archéologique et Historique, CLIV, pp. 231-282.

—, 2002, Problématique et méthodologie archéologiques de la construction de la citadelle de Damas, BerThier S. et EL-AjII E. (eds.), Études et travaux à la citadelle de Damas, 2000-2001: un premier bilan, Bulletin d'études orientales (Damas), 53, 2002, pp. 59-76.

BESSAC J.-C., BRETON J.-F., 2002, Note technique sur la nécropole d'Awâm à Mâ'rib (Yémen), Archäeologische Berichte aus dem Yemen, IX, pp. 117-127, pl. 1-7.
BessaC J.-C., AbDul Massih J., Valat Z., 1997, Enquête ethno-archéologique dans les carrières traditionnelles de Syrie, Doura-Europos, Etudes IV, 1991-1993, Beyrouth, IFAPO, Bibliothèque Archéologique et Historique, CLIX, pp. 159-197.

BESSAC J.-C., PÉCOURT J., 1995, Remarques sur les techniques de construction du second art roman à propos de Saint-André à Souvignargues (Gard), Archéologie du Midi Médiéval, 13, pp. 91-122.

BESSAC J.-C., YASMINE J., 2001, Étude préliminaire sur les chantiers de construction du château de Beaufort, Baal, 4, pp. 241-320.

BoQVIST M., 2002, L'étude archéologique du bâti: l'exemple de la salle à colonnes et ses abords, BERTHIER S. et EL-AjII E. (eds), Études et travaux à la citadelle de Damas, 2000-2001: un premier bilan, Bulletin d'etudes orientales (Damas), 53, pp. 77-78.

DOpéré F., 1997, Données nouvelles pour l'interprétation du vocabulaire des signes utilitaires sur le grès calcareux dans l'architecture gothique brabançonne, Actes du Xe colloque international de glyptographie du Mont-Sainte-Odile (France), 4-9 juillet 1996, Braine-leChâteau (Belgique), Centre International de Recherche Glyptographique, pp. 153-196.

—, 2000, Le chantier de la reconstruction de l'abbaye Notre-Dame à Villers-Devant-Orval (Belgique) au XVIII ${ }^{\mathrm{e}}$ siècle, Actes du XIe colloque international de glyptographie de Palma de Majorque, 2-9 juillet 1998, Braine-le-Château (Belgique) Centre International de Recherches Glyptographiques, pp. 191-223.

Hartmann-Virnich A., 2002, La porte nord de la citadelle de Damas (Bab al Adid), premier rapport de l'étude archéologique des élévations, Berthier S. et El-Aji E. (eds), Études et travaux à la citadelle de Damas, 2000-2001: un premier bilan, Bulletin d'études orientales (Damas), 53, pp. 99-130.

KILL R., 1997, Les signes lapidaires des puits et citernes: présentation d'un thème d'étude, Actes du Xe colloque international de glyptographie du Mont-Sainte-Odile (France), 4-9 juillet 1996, Braine-le-Château, Centre International de Recherches Glyptographiques, pp. 249-281.

Le Mausolée, 1976, Essai de nomenclature des carrières françaises de roches de construction et de décoration, Givors (Rhône), éd. Le Mausolée.

NoËL P., 1968, Technologie de la pierre de taille, Paris. 